\title{
Impulsions nanosecondes de rayons $X$ durs à haut tauX de récurrence et en mode rafale à partir d'un système compact et facilement transportable
}

\author{
A. Khacef, R. Viladrosa, C. Cachoncinlle et J.M. Pouvesle \\ GREMI, CNRS, Université d'Orléans, BP. 6759, 45067 Orléans cedex 2, France
}

\begin{abstract}
Résumé: Les résultats que nous présentons se situent dans la continuité de travaux antérieurs concernant le développement et l'optimisation du générateur X portable SPHINX [1,2]. L'extrapolation de ce générateur à des tensions et des fréquences beaucoup plus élevées, tout en conservant au système son aspect compact, a permis d'obtenir des impulsions très brèves (domaine ns) de photons $X$ durs (jusqu'à $450 \mathrm{keV}$ ) en fonctionnement à très haute cadence suivant deux modes distincts: un mode de fonctionnement prolongé jusqu'à des taux de répétition de $400 \mathrm{~Hz}$ et un mode rafale de quelques tirs à une fréquence de $1 \mathrm{kHz}$.
\end{abstract}

\section{INTRODUCTION}

La production d'impulsions très brèves de photons $\mathrm{X}$ incohérents à partir de systèmes de taille réduite pouvant fonctionner à haute cadence constitue une alternative des plus intéressantes aux générateurs de grande taille disponibles. Longtemps cantonnées à l'observation et à l'étude des phénomènes ultra rapides, ces sources ont trouvé leur champ d'application considérablement élargi ces dernières années.

Dans ce travail, nos recherches ont été essentiellement axées sur le développement et l'optimisation d'un générateur impulsionnel de rayons $X$ durs, fonctionnant à très haute cadence (dans la gamme $100 \mathrm{~Hz}$ $1 \mathrm{kHz}$ ), permettant d'émettre des photons de grande énergie (domaine $>100 \mathrm{keV}$ ) sur des temps très courts (domaine 10-40 ns) avec des doses émises élevées ( $>1 \mathrm{mR}^{*}$ à $1 \mathrm{~m}$ de la source), et qui peut être intégré dans divers systèmes industriels, dans des expériences de laboratoire ou en expérimentation sur site.

\section{PERFORMANCES}

Cette source $\mathrm{X}$ utilise une diode en géométrie de cathode creuse sous basse pression. Le système électrique s'appuie sur un dispositif de mise en forme des d'impulsions THT de type Blumlein associé à un générateur multi-lignes commandé par un "spark-gap" à pression atmosphérique déclenché par une décharge à barrière diélectrique. L'emploi d'un seul commutateur, contrairement à ce qui est fait dans les générateurs de type Marx, est intéressant à plus d'un titre. Ceci nous a permis d'obtenir un ensemble extrêmement compact et facilement transportable. Pour une tension de charge de seulement $35 \mathrm{kV}$, les impulsions de tension de choc ( temps de montée inférieur à 30 ns, FWHM inférieure à $100 \mathrm{~ns}$ ) en circuit ouvert, peuvent atteindre $700 \mathrm{kV}$.

Pour une énergie stockée inférieure à $15 \mathrm{~J}$, ce système a permis d'atteindre à ce jour des doses moyennes supérieures à $3 \mathrm{mR}$ par tir, mesurées à 1 mètre de la source, de photons $\mathrm{X}$ d'énergie comprise entre 5 et $450 \mathrm{keV}$ (la limite inférieure représente la coupure de la fenêtre de sortie de la diode $\mathrm{X}$ ). Suivant les conditions de décharge (pression résiduelle, distance inter-électrodes) la largeur à mi-hauteur des impulsions $X$ est inférieure à 40 ns dans tous les cas. La figure 1 donne un exemple d'évolution temporelle des impulsions ainsi obtenues (détection par diode PIN rapide).

${ }^{*} \mathrm{R}=$ Roentgen: $1 \mathrm{R}=2.58 \times 10^{-4} \mathrm{C} \mathrm{Kg}^{-1}$. 


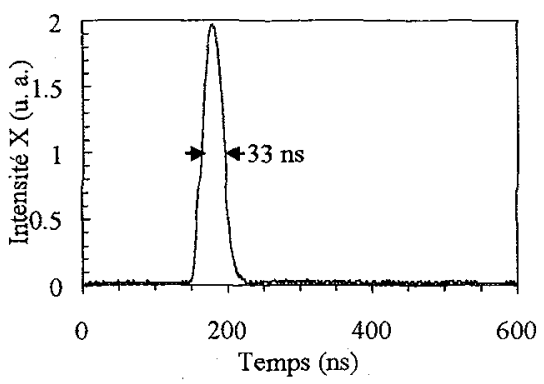

Figure 1: Evolution temporelle de l'impulsion X (Anode tungstène, tension en circuit ouvert $500 \mathrm{kV}$ )

La figure 2(a) montre un exemple des impulsions obtenues quand le système fonctionne en mode prolongé à $400 \mathrm{~Hz}$. Ce taux de répétition de $400 \mathrm{~Hz}$ n'est pas une limite du système lui même mais, il est dû aux performances des alimentations HT utilisées. Pour un fonctionnement sur de longues durées à des cadences aussi élevées, il est nécessaire de prévoir un système de refroidissement de la diode $\mathrm{X}$.

Pour le fonctionnement en mode rafale à très haute cadence, une capacité réservoir $C_{R}$ de valeur comprise entre 0.1 et $2.5 \mu \mathrm{F}$, suivant les conditions de décharge, est utilisée pour le maintien de la charge aux bornes du système de transfert capacitif. Des rafales contrôlées de 2 à 6 tirs à une fréquence de $1 \mathrm{kHz}$ ont été obtenues. La figure 2(b) montre un exemple de résultats obtenus dans le cas d'une rafale de 2 tirs.

Les évolutions temporelles des impulsions $X$ montrées sur la figure 2 ne sont pas représentatives de la durée de celles-ci. En effet ${ }_{2}$ la période entre deux impulsions (domaine ms) est très grande devant la durée d'une impulsion (domaine ns), l'observation du phénomène répétitif à haute fréquence oblige l'utilisation d'un détecteur désadapté.
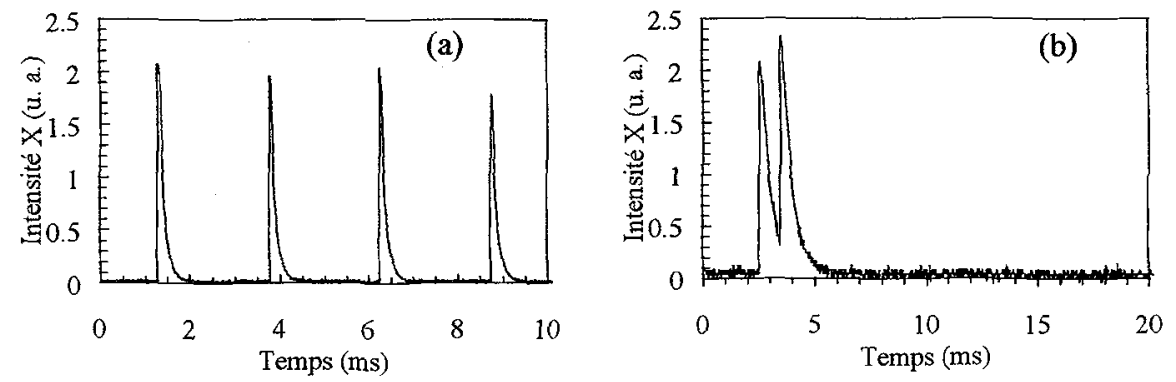

Figure 2: Evolution temporelle des impulsions $X$ : (a) fonctionnement en mode répétitif à $400 \mathrm{~Hz}$, (b) fonctionnement en mode rafale: 2 tirs, $1 \mathrm{kHz}$. (Détecteur désadapté pour permettre la visualisation des impulsions)

\section{Remerciements}

Ce travail a bénéficié du support financier de la DRET convention $N^{\circ} 94.34 .125 .00 .470 .75 .01$

\section{Références}

1. A. Khacef, R. Viladrosa, C. Cachoncinlle, E. Robert, and J. M. Pouvesle, Rev. Sci. Instrum. 68, 2292 (1997)

2. R. Viladrosa, A. Khacef, C. Cachoncinlle, and J. M. Pouvesle, Proc. SPIE 2869, 1086 (1997). 